\title{
REALIDADE AUMENTADA NO DESENVOLVIMENTO DE JOGOS EDUCACIONAIS: UM ESTUDO DE CASO DE UM JOGO DE LÍNGUA INGLESA
}

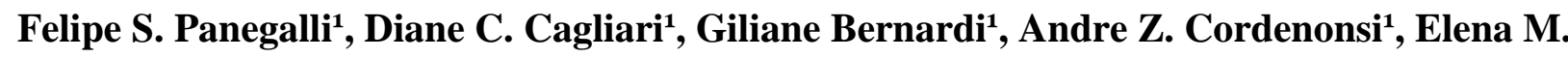 \\ Mallmann' \\ ${ }^{1}$ Mestrado em Tecnologias Educacionais em Rede - Universidade Federal de Santa Maria (UFSM) \\ 97105-900 - Santa Maria - RS - Brasil \\ felipe.panegalli@gmail.com, dianecagliari@gmail.com, giliane@inf.ufsm.br; \\ andrezclinf.ufsm.br; elenamaria@smail.ufsm.br
}

Resumo: A educação vem sendo construída e reconstruída constantemente como uma tentativa de desenvolver novas formas de aprendizagem. Uma dessas tentativas é a Realidade Aumentada (RA) que tem se tornado amplamente utilizada para proporcionar a interação com o mundo virtual através do mundo real, ou seja, objetos ou informações virtuais sendo utilizados no mundo real. Uma vez que a utilização da RA torna-se viável, pode-se estimular e potencializar o aprendizado dos alunos visto que, além de ser atrativa, a tecnologia desperta a curiosidade dos estudantes mais jovens. Por essa razão, este estudo tem por objetivo discutir a utilização de realidade aumentada no desenvolvimento de jogos educacionais tridimensionais para o apoio ao ensino e aprendizagem de cores em Língua Inglesa através da Realidade Aumentada e como integrar o Vuforia SDK no Unity $3 D$

Palavras-chave: Realidade Aumentada, Jogo Educacional, Objeto de Aprendizagem.

Abstract: Education has been constantly built and rebuilt as an attempt to develop new approaches to learning. Augmented Reality (AR) is one of such attempts and it has been widely used to provide interaction with the virtual world through the real world, which means objects or virtual information being used in the real world. Since the use of Augmented Reality is feasible, it may stimulate and enhance the learning process as technology arouses the curiosity of younger students, besides being considered attractive. Therefore, this study aims to discuss the use of $A R$ in the development of $3 D$ educational games as a support to teaching and learning colors in the English language through AR and how to integrate the Vuforia SDK in Unity $3 D$. Keywords: Augmented Reality, Educational Game, Learning Object. 


\section{Introdução}

O uso das Tecnologias da Informação e Comunicação (TICs) com o objetivo de beneficiar a educação e proporcionar a aprendizagem tem sido amplamente estudado. As tecnologias vêm sendo aprimoradas constantemente tanto no que diz respeito aos softwares quanto ao hardware. Tais aperfeiçoamentos engendraram novos computadores que, ao contrário dos anteriores, os quais eram grandes mas apresentavam pouco processamento, tornaram-se menores e com processamentos altíssimos. Sendo assim, essa mudança possibilitou a realização de feitos que eram considerados utópicos até recentemente. Como um exemplo, pode-se citar a inteligência artificial, onde várias equipes trabalham para desenvolver um software capaz de simular o córtex humano (WILD, 2011); e o relógio inteligente, praticamente um computador de pulso, algo inimaginável até o século passado mas que é uma realidade devido ao uso de microcomponentes (PASCOE; THOMSON, 2007).

Dentre muitas possibilidades tecnológicas, nos deparamos com a utilização da Realidade Aumentada (RA) como facilitadora no processo de ensino e aprendizagem. Alguns estudos recentes demonstram a ampla gama de possibilidades geradas pelo uso da RA. Como exemplo, citamos o uso de RA em Drones para simulação de construções, visualizando-as no chão e tomando decisões em tempo real pela empresa (VOLPICELLI, 2015). Ainda, na área de saúde, a RA está crescendo cada vez mais com soluções onde pacientes que possuem limitações físicas ou deficiências possam realizar tarefas simples como enxergar, que é o objetivo do projeto Argus2 (GORDON, 2015), em um caso recente no qual os óculos desenvolvidos pela empresa possibilitaram à mãe ver seu filho recém-nascido pela primeira vez.

Na educação, o uso da RA tem se intensificado nos últimos tempos. Um apontamento que consideramos relevante são os jogos desenvolvidos com fins didáticos (CLAUDIA; MOREIRA, 2013). Já que é crescente o número de alunos que têm acesso à notebooks e smartphones, a RA é apresentada aqui com o propósito de utilizar essas ferramentas em sala de aula como uma forma de apoio à aprendizagem. Este apontamento vai ao encontro do que propomos neste estudo que é promover o desenvolvimento de um jogo educacional para apoio ao ensino e aprendizagem de cores em Língua Inglesa através da Realidade Aumentada e como integrar o Vuforia SDK no Unity3D. Desta forma, busca-se estimular e potencializar o aprendizado dos alunos.

\section{Realidade Aumentada na Educação}


A realidade aumentada surgiu na década de 60 quando Ivan Sutherland desenvolveu o HMD (Head-Mounted Display), um capacete para imergir em um ambiente 3D onde não se poderia distinguir o real do virtual. Porém, com a limitação da tecnologia da época, o HMD só podia ser usado em tempo real por wireframe. Em 1998, Jun Rekimoto apresentou a realidade aumentada através de marcadores 2D que podem ser identificados através de um quadrado parecido com o atual QR Code, onde cada marcação é específica e pode identificar uma quantidade definida de objetos (REKIMOTO, 1998).

Compartilhando a mesma ideia de interação do mundo real com objetos virtuais, Azuma (2001) elenca as propriedades da realidade aumentada, sendo elas: mistura do real e do virtual no ambiente real; interatividade em tempo real; e, por fim, o registro em três dimensões que, conforme o autor, é o alinhamento preciso de objetos reais e virtuais. Conforme Kirner (2011), RA é a mistura de ambos os mundos - virtual e real, ou seja, sobrepor informações virtuais no mundo real (TOSHNIWAL; DASTIDAR 2014). A figura 1 apresenta um exemplo de realidade aumentada.

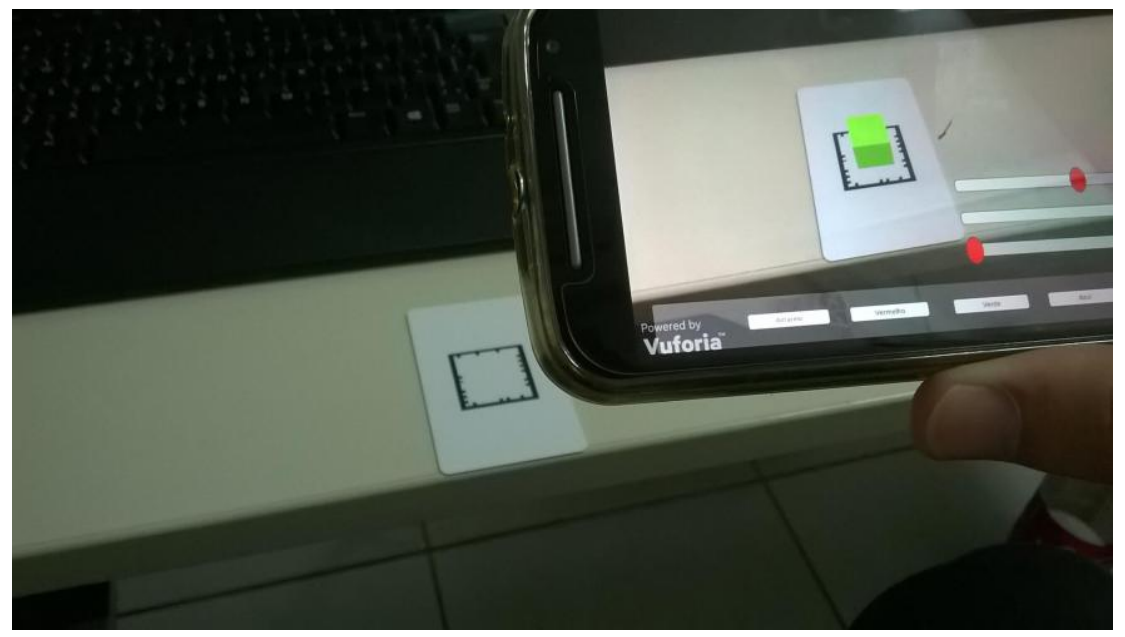

Figura 1 - Realidade Aumentada de um cubo em um ambiente real.

Conforme a Figura 1, podemos analisar o cubo sendo criado através de um marcador 2D que se encontra impresso em um cartão. Como o cubo é um objeto virtual, ele não faz parte do mundo real. No entanto, ao utilizarmos uma ferramenta (câmera do smartphone), ele nos mostra o objeto virtual.

Já que é possível mudar as informações do objeto virtual através da interação com o smartphone, a aplicação se torna interativa, o que pode estimular o aprendizado. Isso é possível 
devido à utilização de ferramentas específicas para a elaboração de aplicações de RA, como por exemplo Metaio ${ }^{1}$, ARToolKit $^{2}$, ARTag $^{3}$, FLARToolKit $^{4}$, VuforiaSDK, entre outros.

Entre as tecnologias que vem sendo fortemente utilizadas no desenvolvimento de aplicações educacionais, com possibilidade de desenvolvimento tridimensional e integração com ferramentas de Realidade Aumentada, destaca-se a engine Unity3D (LEITÃO, 2013), que será utilizada neste trabalho.

O desenvolvimento de aplicações com RA na Unity3D pode ser realizado com a utilização da ferramenta Vuforia, considerada facilmente integrável com a engine, possuindo rápida detecção dos locais de objetos, amplo reconhecimento de marcadores, bem como a qualidade de renderização (Vuforia, 2015). A próxima seção destaca aspectos de desenvolvimento de jogos utilizando Unity3D, bem como a integração com RA por meio da ferramenta Vuforia.

\section{Desenvolvimento de Jogos com Unity3D e Vuforia}

A Unity $3 \mathrm{D}^{5}$ é uma engine de desenvolvimento de jogos 2D/3D, desenvolvida pela Unity Technologies. Com ela, podem ser desenvolvidos diversos gêneros de jogos, sendo eles: educacionais, fps, de plataforma, RPGs, entre outros (UNITY3D, 2015a). O grande crescimento da Unity3D ocorreu pela aceitação do uso da ferramenta que possui suporte com as linguagens mais utilizadas no mercado de desenvolvimento de jogos, tais como C\#, Javascript e Boo; outro aspecto facilitador da sua popularização foi a capacidade de desenvolver para as plataformas: Windows, Linux, Mac, IOS, Wii, Playstation 3 e 4, Xbox 360 e One e Web(através do Unity Web Player) (UNITY 3D, 2015b).

Para desenvolver um jogo com Realidade Aumentada, pode ser utilizado o SDK (Software Development Kit) Vuforia $^{6}$, desenvolvido pela Qualcomm ${ }^{\circledR}$ (VUFORIA, 2015), que consiste em uma extensão para leitura de marcações 2D através de dispositivos que possuem câmera. A integração do Vuforia com o Unity3D é um procedimento considerado simples desde que se tenha alguns cuidados.

\footnotetext{
${ }^{1} \mathrm{http}: / /$ www.metaio.com/

${ }^{2} \mathrm{http}: / / \mathrm{www}$.hitl.washington.edu/artoolkit/

${ }^{3} \mathrm{http}: / / \mathrm{www}$. artag.net/

${ }^{4}$ http://www.libspark.org/wiki/saqoosha/FLARToolKit/en

${ }^{5}$ http://unity3d.com/pt

${ }^{6}$ https://developer.vuforia.com/
} 
Dentro do pacote do Vuforia, quando importado para o Unity3D, existe uma estrutura de pasta que é considerada importante, pois é nela onde ficam armazenados todos os arquivos para trabalharmos a RA e também para identificarmos onde ficam os marcadores que são essenciais para o jogo, pois, conforme Rekimoto (1998), são os marcadores que vão gerar os objetos virtuais no mundo real. De acordo com Leitão (2013), o Vuforia possui uma documentação completa, além de reconhecer qualquer tipo de imagem, não somente marcadores $2 \mathrm{D}$, e também é ágil para o reconhecimento de imagens/marcadores.

\section{Trabalhos Correlatos}

Há na comunidade científica, grande aceitação de trabalhos com realidade aumentada e virtual. Como exemplo, podemos citar diversos simuladores e jogos desenvolvidos para áreas específicas: aeronáutica, construção civil, marketing, educação, entre outros. Para Souza e Kirner (2011), a tecnologia da Realidade Aumentada é considerada especialmente valiosa na área de educação.

Um exemplo destes trabalhos é o Módulo de Geometria Espacial para o Amadeus LMS, desenvolvido na Universidade Federal de Pernambuco. Caracteriza-se por ser um módulo para ensino de Geometria Espacial para educação a distância. De acordo com Rodrigues (2010), o uso da Realidade Aumentada possibilita que o professor mostre aos alunos o objeto geométrico nas diversas perspectivas, fazendo com que o aluno interaja de forma mais dinâmica com a aula de Geometria Espacial.

Outro trabalho envolvendo realidade aumentada é o Vértice Leitão (2013), um jogo de geometria onde o objetivo principal é a compreensão de propriedades dos sólidos geométricos. O jogo trabalha com a dificuldade gradual, isto é, inicia no nível fácil e vai aumentando o grau de dificuldade conforme o jogador evolui no jogo. No jogo, o aluno deve identificar as vértices e arestas. Desta forma, ele vai agregando pontos, elevando sua capacidade cognitiva e liberando conquistas conforme sua pontuação. O jogo foi desenvolvido com softwares gratuitos. Para a modelagem dos sólidos geométricos, utilizou-se o software de modelagem Blender; para a elaboração do jogo foi utilizada a engine Unity3D e, por fim, para a leitura de Realidade Aumentada, foi utilizado o Vuforia.

Magalhães (2010) apresenta um jogo de Realidade Aumentada na área de saúde que possui 3 módulos, sendo eles: um puzzle ou quebra-cabeça anatômico interativo onde o aluno 
monta um esqueleto humano; o Quiz, jogo de perguntas e respostas; e o módulo de Experiência RA onde a partir de marcadores $2 \mathrm{D}$, que podem ser impressos no próprio jogo, o aluno pode visualizar objetos desenvolvidos pelo autor. Na aplicação, foi usada a ferramenta Adobe Flash ${ }^{7}$ com suporte ao ActionScript $3^{8}$. Para a criação dos objetos 3D, foi utilizada a Papervision3D, uma engine de criação de objetos 3D para Flash e o FLARToolkit, uma ferramenta de Realidade Aumentada para integração com a ferramenta Flash.

Com base nos autores citados e por considerar relevante o uso de RA para fins educacionais, foi elaborada a proposta de jogo educacional que será descrita a seguir. Algumas alterações e adaptações ainda podem ser incluídas no jogo conforme o andamento.

\section{Color Challenge - Jogo Educacional desenvolvido com Unity3D e Vuforia}

O ser humano está sempre em busca de descobertas e aprendizados novos através do contato com outras pessoas segundo Dallabona e Mendes (2004). Aprender a língua inglesa é uma dessas formas. Por considerar o lúdico um aspecto significativo no aprendizado de língua inglesa é que foi pensado no desenvolvimento deste jogo educacional.

Nesta seção, será abordado o desenvolvimento de um jogo educacional para apoiar o ensino e aprendizagem das cores em Língua Inglesa utilizando a Unity3D e a ferramenta Vuforia. O jogo denominado Color Challenge pertence ao gênero de perguntas e respostas, onde são adicionados objetos através de marcadores. Com o auxílio de um smartphone, o participante tem que adivinhar qual é a cor daquele objeto. Conforme vai realizando os desafios, o nível de dificuldade aumenta podendo conter mais de um objeto com mais de uma cor, fazendo com que o participante/criança se sinta desafiado e ganhe recompensas conforme vai progredindo. A Figura 2 apresenta como funciona o jogo com a Realidade Aumentada utilizando o smarthphone.

\footnotetext{
${ }^{7}$ https://www.adobe.com/br/products/flash.html

${ }^{8} \mathrm{http} / / / \mathrm{www}$.adobe.com/devnet/actionscript.html
} 


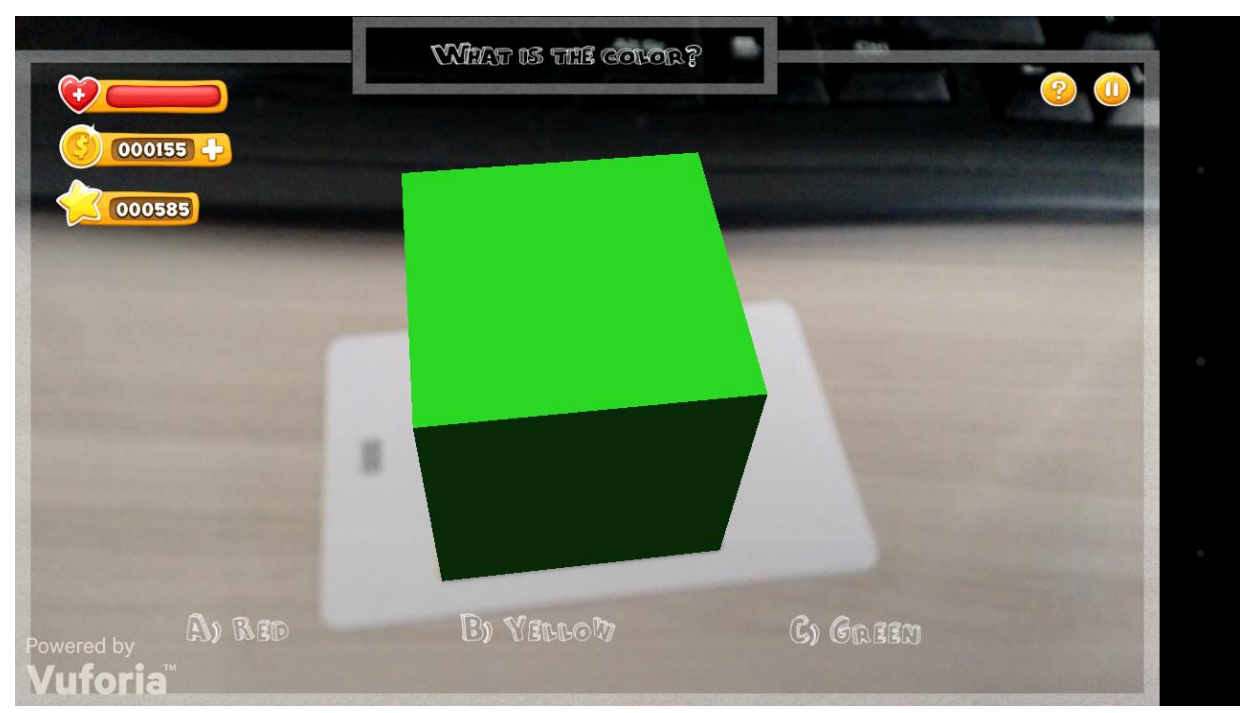

Figura 2 - Jogo Color Challenge com Realidade Aumentada em um ambiente real.

Para que se entenda o funcionamento, é importante ter o auxílio de um professor para iniciar o jogo, o qual é interativo e de fácil compreensão. O jogo possui sons, áudios e objetos voltados para as crianças para que se obtenha maior aceitabilidade e confiabilidade. Nogueira $e t$ al. (2010) afirmam que jogos de computador educativos são formas lúdicas de ensinar as crianças, que aprendem o conteúdo transmitido de uma maneira divertida, interessante, atrativa, eficiente e menos cansativa.

O jogo funciona da seguinte forma: junto com o jogo é disponibilizado um marcador padrão (Figura 3) do Vuforia para mostrar o objeto de Realidade Aumentada.

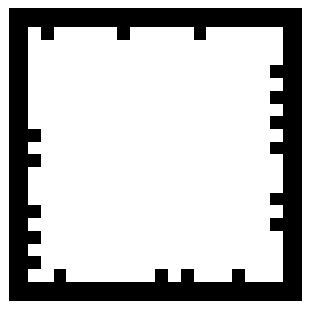

Figura 3 - Marcador padrão utilizado no jogo.

Quando a criança inicia o jogo, ela posiciona o smartphone no marcador para executar o objeto com a cor que, por regra de negócio, troca a cor aleatoriamente e junto com as alternativas que se encontram na parte inferior da tela, sendo que uma das alternativas corresponde a cor gerada no objeto. A tela está dividida em 4 partes sendo elas: no canto superior esquerdo se encontram 3 barras, vida, dinheiro, experiência: a vida é demonstrada em um coração onde a criança pode errar até 3 vezes. Após, o jogo termina e é mostrada a pontuação; as moedas são recompensas ganhas quando se avança de nível. Ganham-se moedas através de experiência: a 
criança acerta as cores e ganha uma quantidade $\mathbf{X}$ de experiência até passar de nível e com isso obtém moedas para comprar power-ups que são dicas ou músicas para o jogo. Na parte inferior, aparecem as alternativas, compostas por A, B e C, sendo duas alternativas erradas e uma certa e por fim o canto superior direito apresenta o botão para pausar o jogo e um de informação para mostrar as instruções do jogo. O jogo destina-se a crianças com idade entre 3 a 4 anos e tem por objetivo, sensibilizá-la para o aprendizado da lingua inglesa.

\section{Considerações Finais}

O objetivo deste artigo foi desenvolver um jogo educacional para apoio ao ensino e aprendizagem de cores em Língua Inglesa, com Realidade Aumentada, utilizando a engine Unity3D e o Vuforia SDK. A relação de escolha dessas ferramentas foram pela fácil integração, além do fato de possuir uma documentação bem elaborada, fazendo com que o desenvolvimento de aplicações de Realidade Aumentada não seja tão árduo de ser elaborado. O "Color Challenge" objetiva sensibilizar as crianças para o aprendizado de língua inglesa, visto que o jogo apresenta uma seleção de vocabulário relacionada às cores, ou seja, palavras fora dos seus contextos, mas que contribuirão com o aprendizado. As crianças são naturalmente curiosas e, por esta razão, o desenvolvimento deste jogo vem ao encontro do que estudiosos como Vigotski (1930) apresentam. Neste caso, a Realidade Aumentada pode ser utilizada como potencializador do processo de aprendizagem.

Para trabalho futuro, será proposta uma avaliação do protótipo desenvolvido junto a um grupo de alunos e também professores de lingua inglesa a fim de avaliar os aspectos como usabilidade e experiência de uso do jogo, além de dar voz aos sujeitos que utilizam o jogo para que melhoramentos possam ser acrescidos. Desta forma, pode-se transformar o protótipo em um jogo operacional por meio de um projeto detalhado, considerando aspectos de engenharia de software e aspectos pedagógicos pertinentes..

\section{Referências Bibliográficas}

AZUMA, R. et al. Recent Advances in Augmented Reality. IEEE Computer Graphics and Applications. v. 21, n. November, p. 1-15, 2001. 
CLAUDIA, L.; MOREIRA, D. S. Expressão gráfica através da realidade aumentada e bim.: uma experiência de visualização. XXI Simpósio Nacional de Geometria Descritiva e Desenho Técnico. Florianópolis, Santa Catarina, 2013.

DALLABONA, Sandra Regina; MENDES, Sueli Maria Schmitt. O lúdico na educação infantil: jogar, brincar, uma forma de educar. Revista de divulgação técnico-científica do ICPG. Vol. 1 n. 4 - jan - mar/2004.

FREIRE, Paulo. Pedagogia da autonomia: saberes necessários à prática educativa. 25 ed. São Paulo. Paz e Terra, 2002.

GORDON, James. The incredible moment a blind mom is able to see her newborn baby son for the very first time using special high-tech glasses. Daily Mail Online, Londres. 24 jan 2015. Disponível em <http://www.dailymail.co.uk/news/article-2924554/The-incredible-moment -blind-mom-able-newborn-baby-son-time-using-special-high-tech-glasses.html>. Acesso em 23 abr. 2015.

KIRNER, C.; KIRNER, T. G. Realidade Virtual e Aumentada: Aplicações e Tendências. Uberlândia: Sociedade Brasileira de Computação, 2011. p.151

LEITÃO, R. M. V. Aprendizagem baseada em jogos: realidade aumentada no ensino de sólidos geométricos. Universidade Aberta, Programa de Pós-Graduação em Expressão Gráfica e Audiovisual, 2013. Dissertação de Mestrado.

MAGAlHÃES, P. S. T. Realidade Aumentada aplicada ao processo de Ensino/Aprendizagem. Instituto Superior de Engenharia do Porto, 2010. Dissertação de Mestrado.

NOGUEIRA, D. et al. Papa Letras: Um Jogo de Auxílio à Alfabetização Infantil. SBGames, p. $170-174,2010$.

PASCOE, J.; THOMSON, K. On the Use of Mobile Tools in Everyday Life Proceedings of the 19th Australasian Conference on Computer-Human Interaction: Entertaining User Interfaces. Anais...: OZCHI' 07.New York, NY, USA: ACM, 2007 Disponível em: <http://doi.acm.org/10.1145/1324892.1324900>. Acesso em: 13 abr. 2015. 
REKIMOTO, J. Matrix: a real-time object identification and registration method for augmented reality. Proceedings. 3rd Asia Pacific Computer Human Interaction (Cat. No.98EX110), 1998.

RODRIGUES, R. L. et al. Realidade Aumentada para o Ensino de Geometria Espacial. Anais do XXI Simpósio Brasileiro de Informática na Educação (SBIE 2010), n. 2005, 2010.

SOUZA, R. C.; KIRNER, C. Ensino e Aprendizagem de Eletromagnetismo usando Recursos de Realidade Aumentada. Novas Tecnologias na Educação, v. 9, 2011.

TOSHNIWAL, R.; DASTIDAR, K. G. Virtual Reality : The Future Interface of Technology. v. 5, n. 6, p. 7032-7034, 2014.

UNITY3D, Technologies. Unity3D Public Relations. 2015b. Disponível em: < https://unity3d.com/pt/public-relations>. Acesso em 02 abr. 2015.

UNITY3D, Technologies. Unity3D User manual. 2015a. Disponível em: < http://docs.unity3d.com/Manual/>. Acesso em 02 abr. 2015.

VIGOTSKI, L. S. (1930). O Desenvolvimento Psicológico na Infância. São Paulo: Martins Fontes, 2003.

VOLPICELli, Gian. Drones That Can See in Augmented Reality. MotherBoard, United Kingdom. 02 abr 2015. Disponível em < http://motherboard.vice.com/read/drones-that-can-seein-augmented-reality>. Acesso em 23 abr. 2015.

VUFORIA, Qualcomm. Manual Vuforia Developer. 2015 Disponível em: <http://developer.vuforia.com/resouces/tutorials/>. Acesso em 28 mar. 2015.

WILD, R. Inteligência Artificial e noções sobre o humano: quem deve saber, quem pode falar. Revista Brasileira de Ciência, Tecnologia e Sociedade, v. 2, n. 1, p. 265-277, 2011. 